\title{
Encoding variability and the concreteness effect in paired-associate learning
}

\author{
RICHARD C. GALBRAITH \\ Northwestern University, Evanston, Illinois 6.0201
}

\begin{abstract}
In an attempt to explain the concreteness effect in PA learning, encoding variability (as measured by contextual variety) was manipulated factorially on the stimulus and response sides within abstract and within concrete noun pairs. The results showed a strong concreteness effect, with increasing encoding variability having no effect on the stimulus side, and a direct positive effect on the response side. The applicability of these results to the encoding-variability hypothesis was discussed.
\end{abstract}

Martin (1968) has proposed that the variability with which a stimulus is encoded can be used as an effective predictor of paired-associate (PA) learning. In particular, the greater the variability of the trial-to-trial stimulus encoding, the smaller the probability that the correct response will be elicited. The measure originally assumed to be representative of encoding variability was the reciprocal of an item's meaningfulness $(M)$ value. That is, as stimulus $M$ decreased, the number of different perceptual encodings to a stimulus was assumed to increase, hence a low-M stimulus was typified as being more variable and less integrated for encoding purposes than a high-M stimulus. The research in this area has up until now been confined mainly to low meaningful trigram stimuli (CCCs, CVCs). The use of words as stimuli has been limited, as the restricted range of word meaningfulness has not lent itself well to experimental manipulation.

In reply to this problem, several authors have recently suggested an alternative measure for an item's encoding variability, one based on the variability of its free-association responses (Butler \& Merikle, 1970; Howe, 1972; Martin, 1972). According to their reasoning, the normative variability of free-association responses to a given item (word) provides a viable indicator of the number of differential perceptions on that item (word) over several presentations. In terms of free-association hierarchies, this would imply that those words having a flat associative hierarchy (characterized by numerous infrequent responses) should be perceived differently more often over repetitions than those having a steep associative hierarchy (composed of a few, frequently repeated responses). Given this new free-association variability measure, then, the encoding variability prediction with high meaningful words would be that PA performance should increase as the free-association variability of the stimulus words decreases.

The following experiment was performed as a test of

Thanks are especially due Benton J. Underwood for his helpful comments on an earier draft of this paper. the encoding variability hypothesis with high meaningful items (words). The measure used here (contextual variety) was based on the judged number of different contexts in which a word had previously been experienced (Galbraith \& Underwood, 1973). The logical extension made here was that a word judged to have occurred in fewer different contexts was also assumed to have a smaller degree of encoding variability. Accordingly, it was assumed that those words rated high in contextual variety should have a greater probability of being encoded differently over repetitions than those words rated low. It may well be that an encodingvariability theorist would not find this measure appropriate. However, the defining operations for contextual variety appear to make the presumed relationship between encoding variability and contextual variety a reasonable one. Contextual variety was manipulated on the stimulus and response sides, with the prediction being that if the variety of a word's prior contextual usage influenced its encoding, then PA performance should decrease as stimulus contextual variety increased.

Since the words used in this experiment were also scaled for concreteness, one further issue lent itself to examination here. A consistent empirical finding in the literature is that increasing concreteness of the stimulus terms facilitates PA learning (e.g., Paivio, 1965). Paivio (1963) has suggested that the underlying mechanism for this concrete stimulus superiority is evoked imagery (of the concrete words). Termed the "conceptual peg" hypothesis, stimulus words are assumed to function as memorial pegs (upon which the responses are "hung" during learning for later recall), with words of higher rated imagery providing the more "solid" pegs. An alternative interpretation to this hypothesis has since been presented, one based on a proposed encoding-variability difference between abstract and concrete words. This alternate hypothesis suggests that the relatively slower learning of abstract words was due to their greater encoding variability (relative to concrete words) over trials (Paivio, 1971, p. 287). This is not unreasonable in light of the substantial negative 
Table 1

Lists Used: Concreteness Values from Paivio, Yuille, and Madigan, 1968; Contextual

Variety Values from Galbraith and Underwood, 1973

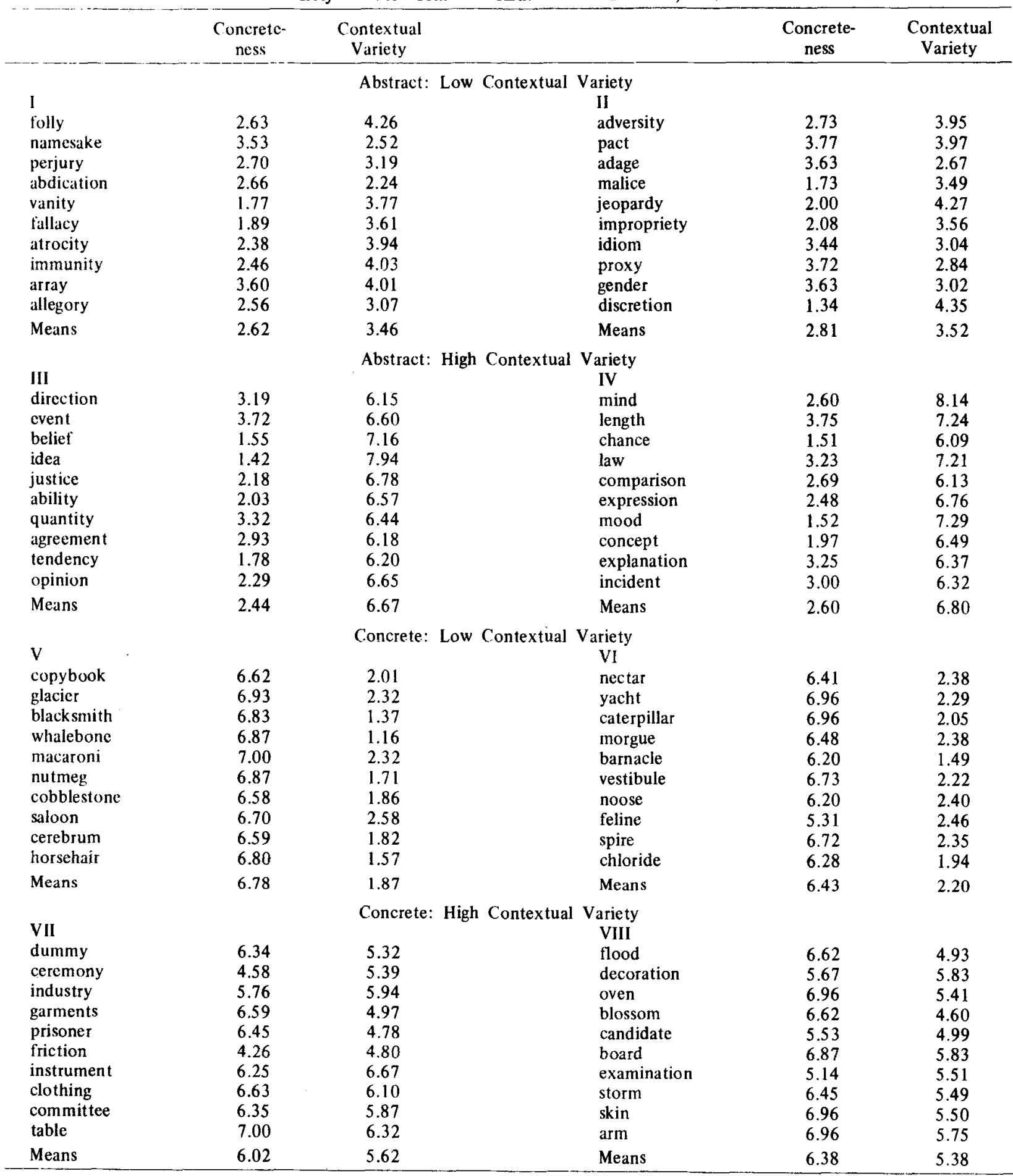

correlation $(\mathrm{r}=-.54)$ found between concreteness and contextual variety (not reported originally in Galbraith \& Underwood, 1973). As a test of the latter hypothesis, the present experiment paired concrete and abstract words of differential contextual variety in a PA task. The prediction resulting from this manipulation was that if the concreteness effect was in fact due to the greater encoding variability of abstract words, then the PA learning difference in favor of the concrete words should be nullified when abstract and concrete words were equated on encoding variability (contextual variety).

In summary, the present experiment was designed as a 
Table 2

Combination of Lists Used

\begin{tabular}{cccc}
\hline \multirow{4}{*}{ Abstract } & List & $\begin{array}{c}\text { Stimulus } \\
\text { Terms }\end{array}$ & $\begin{array}{c}\text { Response } \\
\text { Terms }\end{array}$ \\
\hline \multirow{5}{*}{ Concrete } & L-L & I & II \\
& L-H & I & III \\
& H-L & III & I \\
& H-H & III & IV \\
& L-L & V & VI \\
& L-H & V & VII \\
& H-L & VII & V \\
& H-H & VII & VIII \\
\hline
\end{tabular}

test of the two preceding issues related to the encoding-variability hypothesis. To do this, stimulus and response variability was manipulated factorially within concrete and within abstract noun pairs. As this relates to the concreteness effect in PA learning, if the encoding variability of abstract noun pairs and concrete noun pairs was equated, then the positive concreteness effect found in PA learning should not appear. With respect to the encoding variability of high meaningful words, increases in contextual variety on the stimulus side should produce a negative effect on PA performance within both the abstract and concrete noun pairs.

\section{METHOD}

\section{Materials and Lists}

A total of 80 words was selected from the 158 scaled for contextual variety by Galbraith and Underwood, 1973. These included the top and bottom 20 words from both the abstract and concrete dimensions. Four 10-pair lists, representing the four combinations of stimulus and response variability, were constructed from the 40 abstract and 40 concrete words separately (see Table 1). The actual PA list combinations of low-low (L-L), low-high (L-H), high-low (H-L), and high-high $(\mathrm{H}-\mathrm{H})$ variability are shown in Table 2 . Words having the same ordinal position in the presented lists were paired together in the experiment (e.g., in the concrete L-L list, copybook and nectar were paired together; in the concrete L-H list, copybook and dummy were paired together). As is evident in the experimental lists, no two words having the same first letter were paired. It can also be seen from the scaled contextual variety values in Table 1 that the means for the abstract and concrete noun pairs differed in absolute value. Part of this was due to the fact that concreteness and contextual variety correlated -.54 . These differences and their potential effect on the results will be discussed later.

\section{Subjects}

A total of 96 subjects, 12 in eacin of the eight conditions, served in the experiment. All subjects were enrolled in an introductory psychology course at Northwestern University.

\section{Procedure}

Subjects were tested individually, using a memory drum. The anticipation method was used, with the pairs being presented at a 1.5/1.5-sec rate, with a 3-sec intertrial interval. An, experimental performance criterion required each subject to respond for 10 trials, or until he responded perfectly on three successive trials. The subjects achieving three successive perfect trials prior to 10 trials were stopped, and their remaining trials scored as perfect. The eight conditions (four combinations of contextual variety within both levels of concreteness) were blocked randomized, and subjects were assigned to conditions in order of their appearance at the laboratory.

\section{RESULTS}

The response measure used was the number of correct anticipations per subject per trial. Figure 1 shows the mean performance for the eight groups over the 10 trials. Perhaps the most striking aspect of Figure 1 is the pronounced superiority of the concrete over the abstract noun pairs. Overall, this difference is more than 2 to 1 in favor of the concrete pairs (5.98-2.72), and highly significant statistically, $F(1,88)=105.83, p<.001$. In addition, there was a significant Trials by Concreteness interaction $[F(9,792)=7.11, p<.001]$, indicating a differential increase over trials in favor of the concrete pairs. Since it may be argued that the abstract and concrete pairs were not strictly equated on variability, the performance superiority for the concrete lists may have been due to the overall lower variability of the concrete pairs. A brief inspection of the results, however, shows that the performance of the low variable abstract stimuli does not even approximate the performance of the high variable concrete stimuli. Thus, it can be concluded that the present experimental equating of the encoding variability of the abstract and concrete noun pairs did not neutralize the concreteness effect in PA learning.

It can also be seen from Figure 1 that the effect of stimulus and response encoding variability was contrary to that predicted by the encoding-variability hypothesis. Whereas the manipulation of the encoding variability had no reliable effect on the stimulus terms

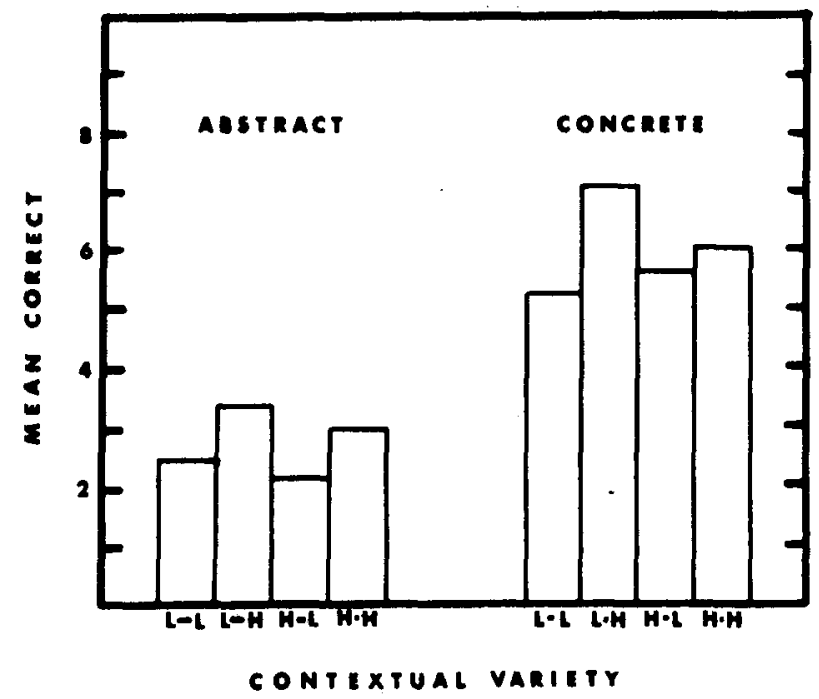

Figure 1. Mean number of correct responses per trial for stimulus-response pairs of low $(\mathrm{L})$ and high $(\mathrm{H})$ contextual variety within abstract and within concrete noun pairs. 
$[F(1,88)=1.30, p>.05]$, it had a direct positive one on the response terms $[F(1,88)=9.27, p<.005]$. Again, one might question these effects since they are based on different absolute levels within the concrete and within the abstract noun pairs. Hence, separate analyses for the effects of stimulus and response variability were conducted both within the concrete and within the abstract noun pairs. These analyses produced the same overall picture of a significant response and a nonsignificant stimulus variability effect within concrete $[F(1,44)=5.22, \quad p<.05 ; \quad F(1,44)=.61, \quad p>.05$, respectively $]$ and within abstract $[F(1,44)=4.07$, $p<.05 ; F(1,44)=.73, p>.05$, respectively]. Thus, in direct opposition to the earlier prediction based on the encoding-variability hypothesis, stimulus variability was not found to have a significant effect, while response variability produced a significant direct effect on the PA learning of the abstract and concrete noun pairs.

\section{DISCUSSION}

These data clearly go against an encoding-variability explanation for the concreteness effect in PA learning. The near equating of encoding variability simply did not remove or even reduce the magnitude of the concreteness superiority. If anything, the present methods appeared to have enhanced this effect. Whereas Paivio (1965) found nearly a 2 to 1 difference (11.41--6.05) by his mixed-list procedure with four trials, the present data showed nearly a 3 to 1 $(4.12-1.42)$ average difference between concrete and abstract noun pairs, with the L-H concrete and H-L abstract pairs showing a 5 to 1 difference $(5.19-1.01)$ over the first four trials. For whatever reason the concreteness effect occurred in these data, the encoding variability interpretation simply did not seem capable of explaining it.

The recent findings of Peterson and McGee (1974) also deny the applicability of the encoding-variability hypothesis in the area of recognition and recall of abstract and concrete nouns. Using the number of dictionary meanings as an index of conceptual complexity (encoding variability?), their results showed conceptual complexity to be incapable of accounting for the effect of concreteness in either recognition or recall learning.

Within each level of concreteness, the manipulation of stimulus encoding variability did not yield results compatible with the encoding-variability hypothesis. Increasing stimulus encoding variability produced no reliable difference on PA performance. Increasing response encoding variability, on the other hand, had a significant direct effect on PA learning. Insofar as contextual variety is deemed an acceptable measure of encoding variability, the present results using high meaningful items (words) did not support the encoding-variability hypothesis of PA learning.
The overall lack of an effect of stimulus encoding variability found in the present study is consistent with the results reported by Paivio (1968). In that same study, however, response encoding variability was found to be negatively correlated with the pooled index of two measures of associative variety (the correlation was small, but significant; $r=-.22$ ). As his experiment employed a mixed-list design including concreteness, associative variety, and stimulus and response combinations, priority effects may have contributed to this finding.

The present results, and in particular those of the concrete noun pairs, are also in conflict with the generality-specificity position of Paivio and Olver (1964). In their experiment, four types of concrete noun pairs were utilized: general-general, general-specific, specific-general, and specific-specific. Although not precisely defined, concrete words having more or less unique referents were considered "specific" (table, dollar), while those of a less distinct nature were considered "general" (furniture, money). Their results showed stimulus specificity to be positively related to recall, but response specificity to be unrelated. However, as this was a mixed-list manipulation, prionty effects may have affected this outcome also.

One further point should be addressed here, as the correlation $(r=.92$ for concrete and abstract words combined) between contextual variety and phenomenal frequency was so high (Galbraith \& Underwood, 1973). One might wonder if what was really demonstrated here was an effect of frequency and not of encoding variability. Two studies, one unpublished (Paivio, 1971, pp. 261-262) and the other published (Paivio \& Madigan, 1970), speak somewhat to this possibility. The results of the former experiment showed slight positive effects of response frequency among concrete nouns, with stimulus and response frequency both having a negative effect among the abstract pairs. The results of the latter showed the effects of frequency to be positive on the response side but negative on the stimulus side within the abstract and concrete noun pairs. However, as both of these experiments used a mixed-list design, judgment must be somewhat reserved. Also, the frequency values in these experiments were from the Thorndike-Lorge (1944) norms, which only correlated .48 with phenomenal frequency (as compared with .92 for contextual variety). It is concluded, therefore, that since no consistent effect of frequency with concrete and abstract words has been shown previously, the current results should not be taken as due to a frequency manipulation.

\section{REFERENCES}

Butler, B. E.. \& Merikle, P. M. Uncertainty and meaning fulness in paired-associate learning. Journal of Verbal Learning and Verbal Behavior, $1970,9,634-641$.

Galbraith, R. C., \& Underwood, B. J. Perceived frequency of 
concrete and abstract words. Memory \& Cognition, 1973, 1, 56-60.

Howe, E. S. Number of different free associates: A general measure of associative meaningfulness. Journal of Verbal Learning and Verbal Behavior, 1972, 11, 18-28.

Martin, E. Stimulus meaningfulness and paired-associate transfer: An encoding variability hypothesis. Psychological Review, $1968,75,421-441$.

Martin. E. Stimulus encoding in learning and transfer. In A. W. Melton and E. Martin (Eds.), Coding processes in human memory. Washington. D.C: Winston, 1972. Pp. 59-84.

Paivio, A. Learning of adjective-noun paired-associates as a function of adjective-noun word order and noun abstractness. Canadian Journal of Psy chology, 1963, 17, 370-379.

Paitio, A. Abstractness, inlagery, and meaningfulness in paired-associate learning. Joumal of Verbal Learning and Verbal Behavior, $1965,4,32-38$.

Paivio, A. A factor-analytic study of word attributes and verbal learning. Journal of Verbal Learning and Verbal Behavior, $1968,7,4149$.

Paivio, A. Imagery and verbal process. New York: Holt, Rinehart, \& Winston, 1971.
Paivio, A., \& Madigan, S. A. Noun imagery and frequency in paired-associate and free recall learning. Canadian Journal of Psychology, 1970, 24, 353-361.

Paivio, A., \& Olver, N. Denotative-generality, imagery, and meaningfulness in paired-associate learning of nouns. Psychonomic Science, 1964, 1, 183-184.

Paivio, A., Yuille, J. C., \& Madigan, S. A. Concreteness, imagery and meaning fulness values for 925 nouns. Journal of Experimental Psychology Monograph Supplement, 1968, 76 (No. 1, Part 2).

Peterson, M. J., \& McGee, S. H. Effects of imagery instructions, imagery ratings, and number of dictionary meanings upon recognition and recall. Journal of Experimental Psychology, $1974,102,1007-1014$.

Thorndike, E. L., \& Lorge. I. The teacher's word book of 30,000 words. New York: Bureau of Publications, Teachers College, 1944.

(Received for publication July 31,1974 ; revision accepted September $6,1974$. ) 